\title{
Synthesis of Novel Pyrimido Pyrimidine and Their Derivatives
}

\author{
Sirsat Shivraj Balajirao*, Jadhav Anilkumar Govindrao, Nilesh B Chavhan \\ P. G. Research Centre, Department of Chemistry, Yeshwant Mahavidyalaya, Nanded, India
}

Email address:

sbs.igm@gmail.com (S. S. Balajirao)

${ }^{*}$ Corresponding author

\section{To cite this article:}

Sirsat Shivraj Balajirao, Jadhav Anilkumar Govindrao, Nilesh B Chavhan. Synthesis of Novel Pyrimido Pyrimidine and Their Derivatives. International Journal of Pharmacy and Chemistry. Vol. 6, No. 1, 2020, pp. 1-5. doi: 10.11648/j.ijpc.20200601.11

Received: March 18, 2020; Accepted: April 1, 2020; Published: April 30, 2020

\begin{abstract}
Chalcone derivatives were synthesis by the reaction of some 4-nitrobenzaldehyde derivatives with acotophenone then the product obtained E-3-(4-nitrophenyl)-1-phenylprop-2-en-1-one. This product is chalcone is another react with guanidine nitrate. The hetrocyclic derivatives of guanidine nitrate respectively the final product have been characterized by elemental analysis. the product obtained is 1,6 dihydro 6(4-nitrophenyl) 4-phenylpyridine 2-amine. In present report novel pyrimidines were prepared from starting materials chalcone and guanidine nitrate. The resulting compound 1,6-dihydro-6-(4nitrophenyl)-4 phenylpyrimidin 2 amine (2) was further reacted with 2 bis(methylthio)methylene malononitrile in the presence of catalytic amount of potassium carbonate in DMF under reflux condition that offered novel 4,6,9,9a-tetrahydro-4-imino-2(methylthio)-8-(4-nitrophenyl) 6 phenyl 1H pyrimido[1,2 a]pyrimidine 3 carbonitrile (3). The synthesized compounds were characterized by spectral methods. The compound (3) possesses replaceable methylthio $\left(-\mathrm{SCH}_{3}\right)$ group at 2 position. The compound (3) react with various nucleophiles like substituted aromatic amines, aromatic phenols, hetryl amines and active methylene compounds to give 4,6,9,9a-tetrahydro-4-imino-2-(methylthio)-8-(4-nitrophenyl)-6-phenyl-1H-pyrimido[1,2a]pyrimidine-3-carbonitrile -carbonitrile in good yields.
\end{abstract}

Keywords: Claisen Schmidt Condensation, 2 bis(methylthio)methylene malononitrile Michael Addition Reaction, Pyrimidine

\section{Introduction}

Pyrimidine is a six membered cyclic compound containing 4 carbon and 2 nitrogen atoms and is pharmacologically inactive but its synthetic derivatives possess an important role in modern medicine. The chemistry of heterocyclic compounds is the most important in the discovery of new drugs. There are large number of synthetic heterocyclic compounds, like pyrrole, pyrrolidine, furan, thiophene, piperidine, pyridine and thiazole having important application and many are important intermediates in synthesis. [1] Among all heterocyclic compounds, pyrimidines are one of the most important heterocycles exhibiting remarkable pharmacological activities because it is an essential constituent of all cells and thus of all living matter. [2] Many simple fused pyrimidines such as purines and pteridines are biologically active by themselves. [3.4] or are essential components of very important naturally occurring substances (i.e., nucleic acids). The presence of a pyrimidine base in cytosine, uracil and thymine, which are the essential building blocks of nucleic acids (DNA and RNA) is one of the possible reasons for their activities. [5] Pyrimidine Pyrimidine ring is found in vitamins like isoalloxazine, vitamin $\mathrm{B} 2$, thiamine, riboflavin $(6,7-$ dimethyl-9-(D-1-ribityl) and folic acid. [6] Pyrimidine nucleus is the also present in barbituric acid and its several derivatives like Veranal which are used as hypnotics. [7] Condensed pyrimidine derivatives have been reported as anti-microbial [8], analgesic, antiviral, anti-inflammatory [9], anti- HIV [10], anti-tubercular [11], anti-tumor [12], antineoplastic [13], anti-malarial [14], diuretic [15], cardiovascular [16] agents and hypnotic drugs for the nervous system [17], calcium-sensing receptor antagonists [18] and also for antagonists of the human A2A adenosine receptor. [19] etc Some pteridine derivatives are also used as anti-leukemic drugs [20], or potassium-conserving diuretics. 
[21] In addition, several quinazoline alkaloids exhibit hypnotic [22, 23], bronchodilatory [24], and antimalarial [25, 26] activity. Some fused thieno[3, 2-d]pyrimidines serve as the anti-allergy drugs, some act as fungicides. Examples of some biologically active pyrimidine derivatives are prazosin, quinethazone, trimethotrexate, folic acid, riboflavin. [27]

\section{Methods}

Melting points were determined in open capillary tubes and are uncorrected. The silica gel $\mathrm{F}_{254}$ plates were used for thin layer chromatography (TLC); the spots were examined under UV light and then developed in an iodine vapor. Column chromatography was performed with silica gel (BDH 100-200 mesh). Solvents were purified according to standard procedures. The spectra were recorded as follows: IR, $\mathrm{KBr}$ pellets, a Perkin-Elmer RX1 FT-IR spectrophotometer; ${ }^{1} \mathrm{H} \mathrm{NMR}, \mathrm{CDCl}_{3}, 200 \mathrm{MHz}$, a Varian Gemini 200 instrument. Elemental analysis was performed on a Heraeus CHN-O rapid analyzer.

\subsection{4,6,9,9a-tetrahydro-4-imino-2-(methylthio)-8-(4- nitrophenyl)-6-phenyl-1H-pyrimido[1,2-a]pyrimidine- 3-carbonitrile}

\section{Step - I}

A solution of $\mathrm{KOH} 50 \%$ is added to an equimolar solution of acetophenone $(0.01 \mathrm{~mole})$ and 4-Nitrobenzaldehyde $(0.01$ mole) in ethanol 95\%; the addition is performed under energetic stirring at room temperature. The reaction is left under stirring for one night and then diluted with water and acidified; the precipitate is separated by filtration and dried under vacuum. They are crystallized by ethanol compound.

Step - II

A mixture of chalcone i.e. (E)-3-(4-nitrophenyl)-1phenylprop-2-en-1-one (2.53 gm,0.01mole), and guanidine nitrate $(0.76 \mathrm{gm} ., 0.01 \mathrm{~mole})$ were dissolved in ethanoic potassium hydroxide solution $(10 \mathrm{ml})$ was heated for $4 \mathrm{hrs}$., then it was poured into cold ice obtained 1,6 dihydro 6 (4 nitrophenyl) 4 phenylpyrimidin 2 amine (2).

Step - III

A mixture of 1,6 dihydro 6 (4 nitrophenyl) 4 phenylpyrimidin 2 amine (2) and 2 bis(methylthio) methylene malononitrile in the presence of catalytic amount of potassium carbonate $(10 \mathrm{mg})$ in DMF was refluxed for 4 hours, the reaction was monitored by TLC. After completion, the reaction mixture was cooled at room temperature then wash with water the extracted with ethyl acetate. The extract was concentrated and the residue was subjected to column chromatography (silica gel, n-hexane-ethyl acetate 8:2) to obtain pure solid compound 4,6,9,9a-tetrahydro-4-imino-2(methylthio)-8-(4-nitrophenyl)-6-phenyl-1H-pyrimido[1,2a]pyrimidine-3-carbonitrile -carbonitrile (3). The compound (3) confirmed by IR, ${ }^{1} \mathrm{H}$ and $\mathrm{C}^{13} \mathrm{NMR}$ and $\mathrm{MS}$ analytical data.<smiles>CC(=O)c1ccccc1</smiles><smiles>O=C(/C=C/c1ccc([N+](=O)[O-])cc1)c1ccccc1</smiles>

(E)-3-(4 nitropheny l)-1-phenylprop-2-en-1-one $\mathrm{NH}_{2} \mathrm{CNHNH}_{2} \mid$ Alc.KOH<smiles>NC1=NC(c2ccccc2)=CC(c2ccc([N+](=O)[O-])cc2)N1</smiles>

(2)

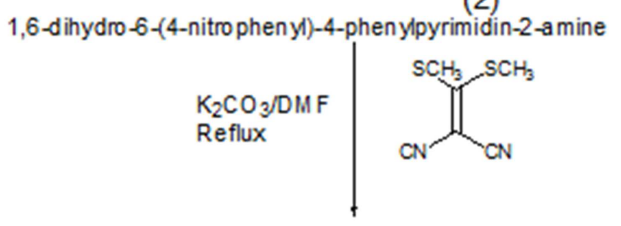<smiles>CSC1=C(C#N)C(=N)N2C(NC(c3ccc([N+](=O)[O-])cc3)=CC2c2ccccc2)N1</smiles>

(3) 


\subsection{Synthesis of Derivatives}

A mixture of (3) (1mmol) and independently, various substituted aromatic amines, aromatic phenols, heteryl amines and active methylene compounds (1mmol) in DMF $(10 \mathrm{ml})$ and anhydrous potassium carbonate $(10 \mathrm{mg})$ was reflux for 4 to $6 \mathrm{hrs}$. The reaction mixture cooled to room temperature and poured into ice cold water. The separated solid product was filtered, washed with water and recrystallised using ethyl alcohol.
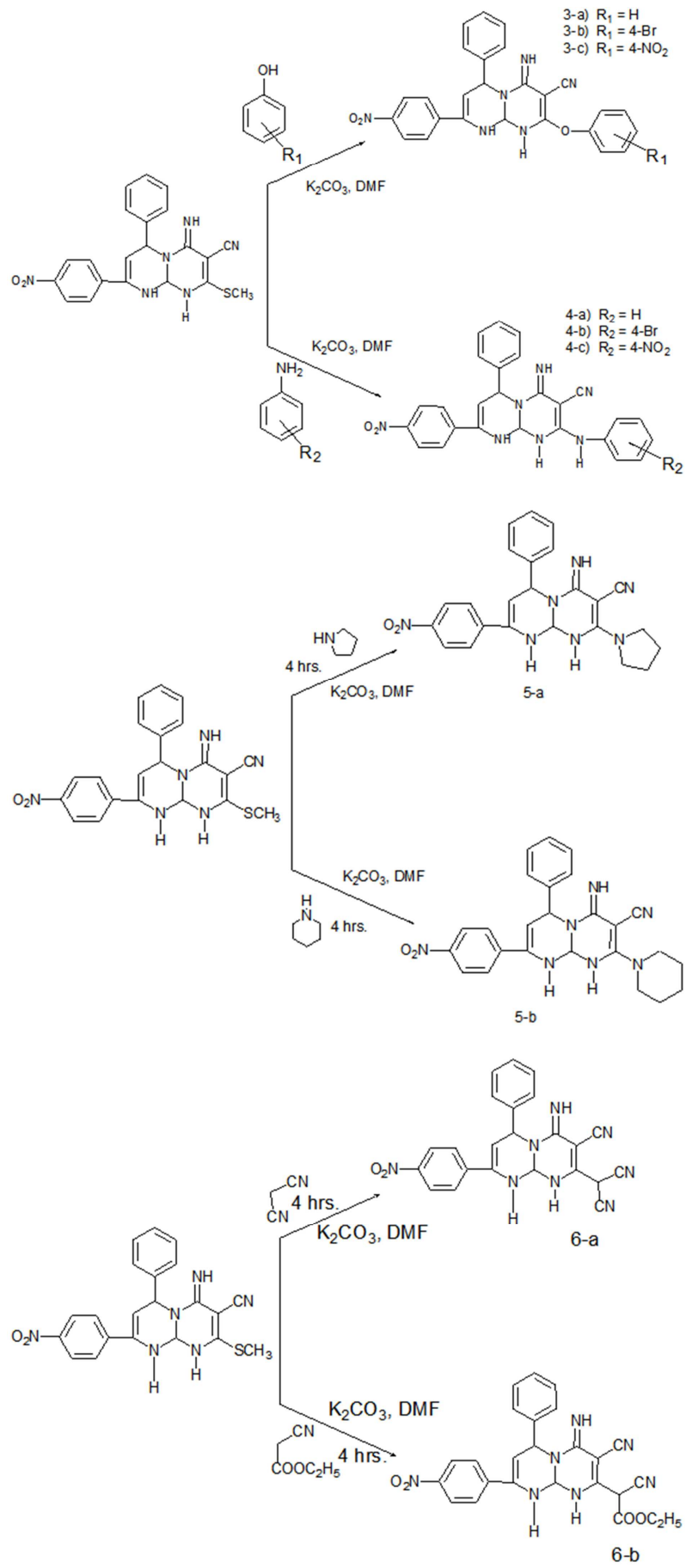

Figure 2. Derivatives of compound no 3.

\section{Result and Discussion}

The compound 4,6,9,9a tetrahydro 4 imino -2(methylthio)- 8- (4 nitrophenyl)-6-phenyl-1H-pyrimido[1,2a]pyrimidine-3-carbonitrile -carbonitrile are synthesized by dissolving 1,6 dihydro 6 (4 nitrophenyl) 4 phenylpyrimidin 2 amine (2) and 2 bis(methylthio) methylene malononitrile in the presence of $\mathrm{K}_{2} \mathrm{CO}_{3}$ in DMF under reflux condition. The synthesized compound acts as electrophilic species reacting with various substituted aromatic amines, aromatic phenols, hetryl amines and active methylene compound gives 4,6,9,9a tetrahydro 4 imino 2 (methylthio) 8 (4 nitrophenyl)-6-phenyl1H-pyrimido[1,2-a]pyrimidine-3-carbonitrile -carbonitrile in good yields.

\section{Conclusion}

A new different 4,6,9,9a-tetrahydro-4-imino-2(methylthio)-8-(4-nitrophenyl)-6-phenyl-1H-pyrimido[1,2a]pyrimidine-3-carbonitrile -carbonitrile are synthesized by using simple and efficient chemistry and this synthesized compounds possesses methylthio group at 8-position which is best leaving group therefore synthesized compound act as an electrophilic species and reacting with various nucleophiles. In compound (3) cyano and thiomethyl groups are at adjacent position it also undergo cyclization to give polycyclic heterocyclic compound.

3) 4,6,9,9a-tetrahydro-4-imino-9-methyl-2-(methylthio)-8(4-nitrophenyl)-6-phenyl-1H-pyrimido[1,2-a]pyrimidine-3carbonitrile.

IR: 3330, 2210, 1650, $1050 \mathrm{~cm}^{-1}$;

${ }^{1} \mathrm{H}$ NMR: $\delta 2.40\left(\mathrm{~s}, 3 \mathrm{H}, \mathrm{SCH}_{3}\right), 5.10(\mathrm{~s}, 1 \mathrm{H} \mathrm{NH}), 5.10(\mathrm{~s}$, $1 \mathrm{H} \mathrm{NH}), 5.15(\mathrm{~s}, 1 \mathrm{H} \mathrm{NH}), 6.48(\mathrm{~s}, 1 \mathrm{H}=\mathrm{CH}) 5.74(\mathrm{~s} 1 \mathrm{H} \mathrm{CH})$, 5.50 (s, 1H CH), 7.18 (s, 5H Ar-H), 7.20 (dd, 2H Ar-H), 6.95 (dd, $2 \mathrm{H} \mathrm{Ar-H),}$

ESI-MS: 418.

Anal.Calcd for $\mathrm{C}_{21} \mathrm{H}_{18} \mathrm{~N}_{6} \mathrm{O}_{2} \mathrm{~S}: \mathrm{C}, 60.27 ; \mathrm{H}, 4.34 ; \mathrm{N}, 20.08$; O, 7.65; S, 7.66

Found: C, 60.27; H, 4.34; N, 20.08; O, 7.65; S, 7.66

Mol. Formula: $\mathrm{C}_{21} \mathrm{H}_{18} \mathrm{~N}_{6} \mathrm{O}_{2} \mathrm{~S}$

Mol.Wt.: 418.

3-a) 4,6,9,9a-tetrahydro-4-imino-8-(4-nitrophenyl)-2phenoxy-6-phenyl-1H-pyrimido[1,2-a]pyrimidine-3-

carbonitrile.

IR: 3330, 2210, 1650, 1050, $\mathrm{cm}^{-1} ;{ }^{1} \mathrm{H}$ NMR: $5.10(\mathrm{~s}, 1 \mathrm{H}$ $\mathrm{NH}), 5.15(\mathrm{~s}, 1 \mathrm{H} \mathrm{NH}), 5.13(\mathrm{~s}, 1 \mathrm{H} \mathrm{NH}), 6.47(\mathrm{~s}, 1 \mathrm{H}=\mathrm{CH})$ $5.75(\mathrm{~s}, 1 \mathrm{H} \mathrm{CH}), 5.52(\mathrm{~s}, 1 \mathrm{HCH}), 7.24(\mathrm{~s}, 5 \mathrm{H} \mathrm{Ar}-\mathrm{H}), 6.90$ (dd, 2H Ar- H), 7.21 (dd, 2H Ar-H), 7.05 (s 5H Ar-H),

ESI-MS: 464. Anal. Calcd.: $\mathrm{C}_{26} \mathrm{H}_{20} \mathrm{~N}_{6} \mathrm{O}_{3} \mathrm{C}, 67.23$; H, 4.34; N, 18.09; O, 10.33

Found: C, 67.23; H, 4.34; N, 18.09; O, 10.33

3-b) 2-(4-bromophenoxy)-4,6,9,9a-tetrahydro-4-imino-8(4-nitrophenyl)-6-phenyl-1H-pyrimido[1,2-a]pyrimidine-3carbonitrile.

IR: 3330, 2210, 1650, 1050, $660 \mathrm{~cm}^{-1}$; ${ }^{1} \mathrm{H}$ NMR:5.11(s,1H $\mathrm{NH}), 5.15(\mathrm{~s}, 1 \mathrm{H} \mathrm{NH}), 5.13(\mathrm{~s}, 1 \mathrm{H} \mathrm{NH}), 6.46(\mathrm{~s}, 1 \mathrm{H}=\mathrm{CH}) 5.73$ 
(s, 1H CH), 5.54 (s, 1H CH), 7.19 (s, 5H Ar-H), 6.94 (dd, 2H Ar-H), 7.23 (dd, 2H Ar-H), 6.88 (dd 2H Ar-H), 7.24 (dd $2 \mathrm{H}$ Ar-H),

ESI-MS:543. Anal. Calcd: $\mathrm{C}_{26} \mathrm{H}_{19} \mathrm{BrN}_{6} \mathrm{O}_{3}$ : C, 57.47; $\mathrm{H}$, 3.52; Br, 14.71; N, 15.47; O, 8.83 Found: C, 57.47; H, 3.52; $\mathrm{Br}, 14.71 ; \mathrm{N}, 15.47 ; \mathrm{O}, 8.83$

3-c) 2-(4-nitrophenoxy)-4,6,9,9a-tetrahydro-4-imino-8-(4nitrophenyl)-6-phenyl-1H-pyrimido[1,2-a]pyrimidine-3carbonitrile.

IR: 3330, 2210, 1650, 1050, $1510 \mathrm{~cm}^{-1}$; ${ }^{1} \mathrm{H}$ NMR: 5.11(s,1H NH), 5.13(s,1H NH), 5.15(s,1H NH), $6.46(\mathrm{~s}, 1 \mathrm{H}$ $=\mathrm{CH}) 5.73(\mathrm{~s}, 1 \mathrm{HCH}), 5.54(\mathrm{~s}, 1 \mathrm{H} \mathrm{CH}), 7.19(\mathrm{~s}, 5 \mathrm{H} \mathrm{Ar}-\mathrm{H})$, 6.94 (dd, 2H Ar-H), 7.23 (dd, 2H Ar-H), 6.88 (dd 2H ArH), 7.24 (dd 2 H Ar-H),

ESI-MS: 509. Anal. Calcd: $\mathrm{C}_{26} \mathrm{H}_{19} \mathrm{~N}_{7} \mathrm{O}_{5}$ : C, 61.29; H, 3.76; N, 19.24; O, 15.70

Found: C, 61.29; H, 3.76; N, 19.24; O, 15.70

4-a) 4,6,9,9a-tetrahydro-4-imino-8-(4-nitrophenyl)-6phenyl-2-(phenylamino)-1H-pyrimido[1,2-a]pyrimidine-3carbonitrile.

IR: 3330, 2210, 1650, 1050, $1590 \mathrm{~cm}^{-1} ;{ }^{1} \mathrm{H}$ NMR: $5.10(\mathrm{~s}$, $1 \mathrm{H} \mathrm{NH}), 4.10(\mathrm{~s}, 1 \mathrm{NH}), 4.19(\mathrm{~s}, 1 \mathrm{NH}), 4.15(\mathrm{~s}, 1 \mathrm{NH}), 6.43$ (s, $1 \mathrm{H}=\mathrm{CH}) 5.75(\mathrm{~s}, 1 \mathrm{H} \mathrm{CH}), 5.54(\mathrm{~s}, 1 \mathrm{H} \mathrm{CH}), 7.20(\mathrm{~s}, 5 \mathrm{H} \mathrm{Ar}-$ H), 6.89 (dd, 2H Ar-H), 7.25 (dd, 2H Ar-H), 7.01 (s 5H Ar$\mathrm{H})$,

ESI-MS: 477. Anal. Calcd: $\mathrm{C}_{26} \mathrm{H}_{21} \mathrm{~N}_{7} \mathrm{O}_{2}$ : C, 67.38; H, 4.57; N, 21.15; O, 6.90

Found: C, 67.38; H, 4.57; N, 21.15; O, 6.90

4-b) 2-(4-bromophenylamino)-4,6,9,9a-tetrahydro-4imino-8-(4-nitrophenyl)-6-phenyl-1H-pyrimido[1,2a]pyrimidine-3-carbonitrile.

IR: 3330, 2210, 1650, 1050, $660 \mathrm{~cm}^{-1} ;{ }^{1} \mathrm{H}$ NMR: $5.08(\mathrm{~s}$, $1 \mathrm{H} \mathrm{NH}), 5.15(\mathrm{~s}, 1 \mathrm{H} \mathrm{NH}), 5.15(\mathrm{~s}, 1 \mathrm{H} \mathrm{NH}), 4.13(\mathrm{~s}, 1 \mathrm{NH})$, $6.46(\mathrm{~s}, 1 \mathrm{H}=\mathrm{CH}) 5.76(\mathrm{~s}, 1 \mathrm{H} \mathrm{CH}), 5.50(\mathrm{~s}, 1 \mathrm{H} \mathrm{CH}), 7.22(\mathrm{~s}$, 5H Ar-H), 6.92 (dd, 2H Ar-H), 7.23 (dd, 2H Ar-H), 6.49 (ddAr-H), 7.32 (dd 2 H Ar-H) ESI-MS: 542 Anal. Calcd for $\mathrm{C}_{26} \mathrm{H}_{20} \mathrm{BrN}_{7} \mathrm{O}_{2}$ : C, 57.57; H, 3.72; $\mathrm{Br}, 14.73 ; \mathrm{N}, 18.08$; O, 5.90 Found: C, 57.57; H, 3.72; Br, 14.73; N, 18.08; O, 5.90

4-c) 2-(4-nitrophenylamino)-4,6,9,9a-tetrahydro-4-imino8-(4-nitrophenyl)-6-phenyl-1H-pyrimido[1,2-a]pyrimidine-3carbonitrile.

IR: 3330, 2210, 1650, 1050, $1580 \mathrm{~cm}^{-1} ;{ }^{1} \mathrm{H}$ NMR: $5.08(\mathrm{~s}$, $1 \mathrm{H} \mathrm{NH}), 5.13(\mathrm{~s}, 1 \mathrm{H} \mathrm{NH}), 5.15(\mathrm{~s}, 1 \mathrm{H} \mathrm{NH}) 4.13(\mathrm{~s}, 1 \mathrm{NH})$, $6.46(\mathrm{~s}, 1 \mathrm{H}=\mathrm{CH}) 5.76(\mathrm{~s}, 1 \mathrm{H} \mathrm{CH}), 5.50(\mathrm{~s}, 1 \mathrm{H} \mathrm{CH}), 7.22(\mathrm{~s}$, 5H Ar-H), 6.92 (dd, 2H Ar-H), 7.23 (dd, 2H Ar-H), 6.49 (dd2H Ar-H), 7.32 (dd $2 \mathrm{H} \mathrm{Ar-H})$ ESI-MS: 508. Anal. Calcd: $\mathrm{C}_{26} \mathrm{H}_{20} \mathrm{~N}_{8} \mathrm{O}_{4}: \mathrm{C}, 61.41 ; \mathrm{H}, 3.96 ; \mathrm{N}, 22.04 ; \mathrm{O}, 12.59$ Found: C, 61.41; H, 3.96; N, 22.04; O, 12.59

5-a) 4,6,9,9a-tetrahydro-4-imino-8-(4-nitrophenyl)-6phenyl-2-(pyrrolidin-1-yl)-1H-pyrimido[1,2-a]pyrimidine-3carbonitrile:

IR: 3330, 2210, 1650, 1050, $1580 \mathrm{~cm}^{-1} ;{ }^{1} \mathrm{H}$ NMR: $5.10(\mathrm{~s}$, $1 \mathrm{H} \mathrm{NH}), 5.15(\mathrm{~s}, 1 \mathrm{H} \mathrm{NH}), 5.18(\mathrm{~s}, 1 \mathrm{H} \mathrm{NH}), 6.45(\mathrm{~s}, 1 \mathrm{H}=\mathrm{CH})$ $5.76(\mathrm{~s}, 1 \mathrm{H} \mathrm{CH}), 5.70(\mathrm{~s}, 1 \mathrm{H} \mathrm{CH}), 7.22(\mathrm{~s}, 5 \mathrm{H} \mathrm{Ar}-\mathrm{H}), 6.90$ (dd,2H Ar-H), 7.23 (dd, 2H Ar-H), 2.60 (t, 4H), 1.65 (m, 4H) ESI-MS:441.
Anal. Calcd: $\mathrm{C}_{24} \mathrm{H}_{23} \mathrm{~N}_{7} \mathrm{O}_{2}, \mathrm{C}, 65.29 ; \mathrm{H}, 5.25 ; \mathrm{N}, 22.21$; O, 7.25 Found: C, 65.29; H, 5.25; N, 22.21; O, 7.25

5-b) 4,6,9,9a-tetrahydro-4-imino-8-(4-nitrophenyl)-6phenyl-2-(piperidin-1-yl)-1H-pyrimido[1,2-a]pyrimidine-3carbonitrile:

IR: 3330, 2210, 1650, 1050, $1580 \mathrm{~cm}^{-1} ;{ }^{1} \mathrm{H}$ NMR: $5.10(\mathrm{~s}$, $1 \mathrm{H} \mathrm{NH}), 5.13(\mathrm{~s}, 1 \mathrm{H} \mathrm{NH}), 5.17(\mathrm{~s}, 1 \mathrm{H} \mathrm{NH}), 6.48(\mathrm{~s}, 1 \mathrm{H}=\mathrm{CH})$ $5.72(\mathrm{~s}, 1 \mathrm{H} \mathrm{CH}), 5.54(\mathrm{~s}, 1 \mathrm{H} \mathrm{CH}), 7.20(\mathrm{~s}, 5 \mathrm{H} \mathrm{Ar}-\mathrm{H}), 6.86$ (dd, 2H Ar-H), 7.23 (dd, 2H Ar-H), $3.13(\mathrm{t}, 4 \mathrm{H}), 1.53(\mathrm{~m}, 6 \mathrm{H})$

ESI-MS: 455. Anal. Calcd: $\mathrm{C}_{25} \mathrm{H}_{25} \mathrm{~N}_{7} \mathrm{O}_{2} \mathrm{C}, 65.92 ; \mathrm{H}, 5.53$; N, 21.52; O, 7.02

Found: C, 65.92; H, 5.53; N, 21.52; O, 7.02

6-a) 2-(dicyanomethyl)-4,6,9,9a-tetrahydro-8-(4nitrophenyl)-4-oxo-6-phenyl-1H-pyrimido[1,2-a]pyrimidine3-carbonitrile

IR: 3330, 2210, 1650, $1050 \mathrm{~cm}^{-1} ;{ }^{1} \mathrm{H}$ NMR:5.10 (s, 1H $\mathrm{NH}), 5.15(\mathrm{~s}, 1 \mathrm{H} \mathrm{NH}), 5.18(\mathrm{~s}, 1 \mathrm{H} \mathrm{NH}), 6.49(\mathrm{~s}, 1 \mathrm{H}=\mathrm{CH}) 5.73$ (s, 1H CH), 5.51 (s, 1H CH), 7.23 (s, 5H Ar-H), 6.91 (dd, 2H Ar-H), 7.22 (dd, 2H Ar-H), ESI-MS: 437. Anal. Calcd: $\mathrm{C}_{23} \mathrm{H}_{15} \mathrm{~N}_{7} \mathrm{O}_{3}, \mathrm{C}, 63.15 ; \mathrm{H}, 3.46 ; \mathrm{N}, 22.42 ; \mathrm{O}, 10.97$ Found:C, 63.15; H, 3.46; N, 22.42; O, 10.97

6-b) ethyl 2-cyano-2-(7-cyano-4,6,9,9a-tetrahydro-2-(4nitrophenyl)-6-oxo-4-phenyl-1H-pyrimido[1,2-a]pyrimidin8-yl)acetate:

IR: $3330,2210,1650,1050,2910,1710 \mathrm{~cm}^{-1} ;{ }^{1} \mathrm{H}$ NMR: $5.08(\mathrm{~s}, 1 \mathrm{H} \mathrm{NH}), 5.15(\mathrm{~s}, 1 \mathrm{H} \mathrm{NH}), 5.11(\mathrm{~s}, 1 \mathrm{H} \mathrm{NH}), 6.44(\mathrm{~s}$, $1 \mathrm{H}=\mathrm{CH}), 5.76(\mathrm{~s}, 1 \mathrm{H} \mathrm{CH}), 5.51(\mathrm{~s}, 1 \mathrm{H} \mathrm{CH}), 7.27(\mathrm{~s}, 5 \mathrm{H} \mathrm{Ar}-$ H), 6.89 (dd, 2H Ar- H), 7.22 (dd, 2H Ar-H),3.98 (s,1H act.$\mathrm{CH}), 4.19$ (q, 2H), 1.22 (t3H). ESI-MS: 484. Anal. Calcd: $\mathrm{C}_{25} \mathrm{H}_{21} \mathrm{~N}_{6} \mathrm{O}_{5}: \mathrm{C}, 61.98 ; \mathrm{H}, 4.16 ; \mathrm{N}, 17.35 ; \mathrm{O}, 16.51$ Found: C, 61.98; H, 4.16; N, 17.35; O, 16.51

\section{Acknowledgements}

The authors are grateful to Dr. G. N. Shinde, Principal, Yeshwant Mahavidyalaya, Nanded. For providing laboratory facilities and Vishnu Chemical Ltd, Hyderabad, for providing spectral data.

\section{References}

[1] Devprakash, AB Udaykumar, Journal of Pharmaceutical Research, 2011; 4 (7): 2436-2440.

[2] T. Sasada, F. Kobayashi, N. Sakai, T. Konakahara, Organic Letters, 2009; 11: 2161-2164.

[3] FL Rodney, G Charles, Skinner, S William, Canadian Journal of Chemistry, 1967; 45: 2213-2216.

[4] VP Litvinov, Advances in Heterocyclic Chemistry, vol. 92, Zelinsky Institute of Organic chemistry, Russian Academy of Sciences, 119991 Moscow, Russia, 2006; 83.

[5] Amir M, Javed SA and Kumar H; Indian J. Pharm. Sciences, 2007; 69 (3): 337-343.

[6] O Stanisaw, (2009), some biologically active pyrimidine derivatives are prazosin, quinethazone trimethotrexate, folic acid, riboflavin, Jord. J. Chem, 4: 1-15. 
[7] Jain MK, Sharnevas SC; Organic Chem.; 2008; 3; 997-999.

[8] Desai K, Patel R, Chikhalia K. J Ind Chem, 2006; 45: 773778 .

[9] Amr EA, Nermien MS, Abdulla MM. Monatsh Chem, 2007; 138: $699-707$.

[10] Fujiwara N, Nakajima T, Ueda Y, Fujita HK, Awakami H. Bioorg Med Chem, 2008; 16: 9804-9816.

[11] Ballell L, Field RA, Chung GAC, Young RJ. Bioorg Med Chem Lett, 2007; 17: 1736-1740.

[12] Wagner E, Al-Kadasi K, Zimecki M, Sawka-Dobrowolska W. Eur J Med Chem, 2008; 43: 2498-2504.

[13] Jean-Damien C, David B, Ronald K, Julian G, Pan L, Robert D. Vertex Pharmaceuticals Incorporated, USA, PCT Int. Appl. 2002; 22: 608 .

[14] Gorlitzer K, Herbig S, Walter RD. Pharmazie, 1997; 52: 670672 .

[15] Ukrainets IV, Tugaibei IA, Bereznykova NL, Karvechenko VN, Turov AV. Khimiya Geterotsiklicheskikh Soedinenii, 2008; 5: 718-729.

[16] Kurono M, Hayashi M, Miura K, Isogawa Y, Sawai K, Kokai Tokkyo Koho JP 1987; 62: 267-272, Chem. Abstr, 1988; 109: 37832 .

[17] Wang SQ, Fang L, Liu XJ, Zhao K. Chinese Chem Lett, 2004; 15: 885-888.
[18] Yang W, Ruan Z, Wang Y, Van Kirk K, Ma Z, Arey BJ. et al. J Med Chem, 2009; 52: 1204-1208.

[19] Gillespie RJ, Bamford SJ, Botting R, Comer M, Denny S, et al. J Med Chem, 2009; 52: 33-47

[20] A Hausen, D Fuchs, G Reibnegger, H Wachter, Cancer, 1984, 53 (7), 1634-6.

[21] T Netzer, F Ullrich, H Priewer, M Majewski, E Mutschler, Brit. J. Pharmac, 1992; 106 (1): 222-226.

[22] Y Zheng, M Sun, Y Liu, M Li, M Ji, Med. Chem, 2011; 7 (4): 295-300.

[23] K Sushil, Kashaw, G Vivek, K Varsha, P Mishra, JP Stables, NK Jain, Med. Chem. Research, 2010; 19: 250-261.

[24] DW Combs, MS Rampulla, RK Russell, RA Rampulla, DH Klaubert, D Ritchie, AS Meeks, T Kirchner, Drug Design Delivery, 1990; 6 (4): 241-254.

[25] AR Katritzky, CW Rees, EFV Scriven, Comprehensive Heterocyclic Chemistry II, Boulton, A. J., Ed., 6, Pergamon Press: Oxford - New York - Tokyo, 1996; 195-231.

[26] G Jian, Z Quan, ON Michael, O Nicanor, A Arba, G Lucia, AJ Lin, Antimicrobial Agents and Chemotherapy, 2005; 49: 4928-4933.

[27] O Stanisaw, Jord. J. Chem, 2009; 4: 1-15. 